

\title{
Exploring the views of desk-based office workers and their employers' beliefs regarding strategies to reduce occupational sedentary behaviour, with an emphasis on technology-supported strategies
}

Stephenson, A., McDonough, S., Murphy, M., Nugent, C., Wilson, I., \& Mair, J. (2020). Exploring the views of desk-based office workers and their employers' beliefs regarding strategies to reduce occupational sedentary behaviour, with an emphasis on technology-supported strategies. JOURNAL OF OCCUPATIONAL AND ENVIRONMENTAL MEDICINE, 62(2), 149-155. https://doi.org/10.1097/JOM.0000000000001777

Published in:

JOURNAL OF OCCUPATIONAL AND ENVIRONMENTAL MEDICINE

Document Version:

Peer reviewed version

Queen's University Belfast - Research Portal:

Link to publication record in Queen's University Belfast Research Portal

\section{Publisher rights}

Copyright 2020 Lippincott, Williams \& Wilkins. This work is made available online in accordance with the publisher's policies. Please refer to any applicable terms of use of the publisher.

\section{General rights}

Copyright for the publications made accessible via the Queen's University Belfast Research Portal is retained by the author(s) and / or other copyright owners and it is a condition of accessing these publications that users recognise and abide by the legal requirements associated with these rights.

Take down policy

The Research Portal is Queen's institutional repository that provides access to Queen's research output. Every effort has been made to ensure that content in the Research Portal does not infringe any person's rights, or applicable UK laws. If you discover content in the

Research Portal that you believe breaches copyright or violates any law, please contact openaccess@qub.ac.uk. 


\section{Running title:}

Reducing office sitting using technology-supported strategies

\section{Main title}

Exploring the views of desk-based office workers and their employers' beliefs regarding strategies to reduce occupational sitting time, with an emphasis on technology-supported strategies.

\section{OBJECTIVE:}

Employee and employer views regarding how technology-supported strategies can best meet their needs to reduce occupational sitting are not well known. This study explored target user and key stakeholder beliefs regarding strategies to reduce occupational sitting focusing on technology-supported approaches.

\section{METHODS:}

Nine focus groups and two interviews (employees, $n=27$; employers, $n=19$; board members, $\mathrm{n}=2$ ) were conducted, transcribed and analysed thematically.

\section{RESULTS:}

The main barrier to reducing sitting was job-related tasks taking primary priority. Intervention designers should consider individual preferences, environmental factors, judgemental culture, productivity concerns and staff knowledge. Technology-supported strategies such as smartphone applications, computer software, wearables and emails were deemed to be useful tools to provide prompts and allow behavioural self-monitoring in an easily individualised manner.

\section{CONCLUSIONS:}

Technology-supported strategies were seen to be valuable approaches and might fruitfully be incorporated into future interventions to reduce sitting time. 
Keywords: Sitting time, Office workers, Focus group, Qualitative Research, Technologysupported strategies, Digital technology

\section{Background}

Sitting based activities have shown to comprise $65-82 \%$ of time at work in industrialised countries ${ }^{1}$ with a large proportion (54-77\%) of office workers' total daily sitting time occurring during their working day. ${ }^{2} 34$ This high occupational exposure to sitting may have broad implications for population health ${ }^{5}$ as recent systematic reviews have found evidence linking it to all-cause mortality, cardiovascular disease, type 2 diabetes, metabolic syndrome and some cancers. ${ }^{67}$ In addition, the relative risks associated with sedentary time are higher among people who are not regularly physically active. ${ }^{89}$ Specifically relating to occupational sitting time, other systematic reviews show associations between occupational sitting and body mass index, diabetes, all-cause mortality and certain cancers. ${ }^{10}{ }^{11}$ Therefore, occupational sitting has become an emergent workplace health issue. ${ }^{12}$

Technology-supported strategies have potential to be a low-cost, high-reach, effective and acceptable way to bring about behaviour change in healthcare interventions. ${ }^{13} \mathrm{~A}$ number of studies exploring technology-supported strategies such as activity trackers, ${ }^{14}$ computer prompts $^{15}$ and smartphones ${ }^{16}$ to reduce sitting time have demonstrated promising results. $\mathrm{A}$ recent systematic review and meta-analysis reported that interventions using computer, mobile or wearable technology can reduce workplace sitting time by $40 \mathrm{~min} /$ workday. ${ }^{17}$

Employee and employer views regarding how technology-supported strategies can best meet their needs to reduce occupational sitting are not well known. Qualitative research allows consultation with the target population and key stakeholders to determine intervention needs and design, 1819 and taking their views on barriers, facilitators, and strategies into consideration may make an intervention more likely to succeed. ${ }^{20}{ }^{21}$ As such, in the workplace setting, there is a need to take into account the views of employers and company board members, alongside those of the employees. ${ }^{22}$

A small number of qualitative studies have explored employee beliefs regarding reducing occupational sitting, ${ }^{42324}$ however, none have consulted managers at company board level. 
Participants in these studies mentioned technology-supported strategies such as activity trackers, smartphone applications (apps) and digital prompts as potentially feasible strategies, ${ }^{424}$ however, these were not the focus of these discussions.

There is little evidence exploring employee perceptions of how these types of digital tools may be received as an intervention to reduce occupational sitting time. Furthermore, even less research has investigated how key stakeholders perceive technology-supported strategies as a workplace intervention approach. The current study sought to address these gaps by exploring the perceptions of employees, employers and company board members on their beliefs regarding implementable strategies with an emphasis on technology-supported strategies to best meet the needs of desk-based office workers and their employers in order to reduce sitting time.

\section{Methods}

Ethical approval was granted by Ulster University's School of Sport filter committee. Information sheets were sent to three private and two public organisations in Northern Ireland, identified based on the researchers' networks. In each organisation, a contact person identified potential participants. Those interested were provided with information sheets and given at least 48 hours to reflect on study participation.

Employees were recruited if they met the following inclusion criteria; desk-based office workers aged 18-65 years, working at least three days per week and fluent in English. Employees were excluded if they were non-ambulatory or pregnant. Employers and board members had to be aged 18- 65 years, fluent in English, business owners, line managers, responsible for organisational occupational health and safety, or members of the board of directors from the company/organisation.

It was not possible to conduct focus groups with board members due to a smaller pool from which to recruit and scheduling conflicts, therefore individual interviews were used. Data collection took place at the respective workplaces. Employee, employer and board member focus groups/interviews were conducted separately to avoid group hierarchies. Prior to the 
focus group/interview, written informed consent was obtained and participants completed a demographics questionnaire.

The questioning routes for all groups centred on attitudes toward workplace sitting time, effects on productivity, difficulties in reducing sitting time and approaches to reduce sitting time with an emphasis on technology-supported strategies. In addition to this, the employers and board members were asked about their views on employee sitting and implementation barriers to reducing sitting time (supplementary file 1). The content of the topic guide was based upon the research team's academic experience and components of the Theoretical Domains Framework (TDF) (supplementary file 2). ${ }^{25} 26$ Questions and probes were designed to generate discussion within the TDF domains such as "Environmental Context and Resources", "Social Influences" and "Beliefs about Consequences", as detailed in supplementary file 1.

The topic guide was piloted with a group of desk-based employees $(n=8)$ and was improved upon iteratively. This data was not included in the analysis. All discussions were facilitated by a moderator to direct and maintain the discussion. The moderator explained the guidelines, set ground rules and introduced the concept and consequences of high levels of sitting time. The questioning was semi-structured and open-ended to encourage discussion, whilst probes were used to solicit additional information when required.

Descriptive analysis of the demographic data was conducted using SPSS 23.0. The audiotapes were transcribed verbatim. Anonymity was assured by removing identifiable information from the transcripts. Thematic analysis was used to systematically identify, organise, and offer insights into patterns of meaning i.e. themes. ${ }^{27}$ Each transcript was read independently several times by two members of the research team. Coding was undertaken independently, to assign conceptual labels to relevant parts. After both researchers had separately identified initial codes, they met to discuss until consensus was reached. The codes were sorted into potential themes. This led to the development of a thematic framework which was iteratively refined to reflect emerging views, with constant moving back and forward between the entire data set, the coded extracts and the themes being produced. ${ }^{27}$ Consensus was reached on the 
finalised themes by three members of the team. Quotes were selected to characterise each theme.

\section{Results}

Five focus groups were conducted with employees ( $n=27,33 \pm 12.3$ years old, $37 \%$ female), four focus groups with employers ( $n=19,44 \pm 8.6$ years old, $42 \%$ female) and two interviews with board members ( $n=2,50 \%$ female). The companies involved consisted of an architectural firm, medical equipment developers, technology company, district council and a regional governmental department. A range of roles were represented including architects, engineers, receptionists, and software developers. None of these companies had implemented formalised approaches to reducing sitting time. Employees self-reported sitting for 6.7 hours \pm 0.9 at work each day. This was measured as part of a questionnaire designed for this study, by asking each employee "How many hours per day do you spend sitting at work (on average)?". The focus groups/interviews ranged in length from 23 to 56 minutes with a mean duration of 43 minutes. For further information on participant characteristics, see Supplementary File 3.

\section{Themes}

The main theme to emerge was the 'primacy of work'. Employees, employers and board members felt that while at work, the employees' main priority was to complete job tasks and meet business objectives. Four further themes were identified which captured the barriers and facilitators to reducing employee sitting. These themes and their associated participant quotes are displayed in Table 1.

INSERT TABLE 1

\section{Environment}

In general, participants reported that the office design was centred around sitting. All staff involved in this study used a seated workstation and did not have access to a sit-stand work desk (SSWD). This inadaptable seated environment was viewed as a major barrier to reducing sitting time. Most participants showed an interest in height adjustable furniture and agreed 
that having access would encourage them to reduce sitting time. It was noted that adjustable furniture is costly and may not be used in the long term.

Participants recognised that they felt "at home" while at their desks and this limited incidental opportunities for physical activity (PA) as they had all requirements to hand. In contrast, others saw office design as a facilitator to reduce sitting time. They pointed out that a centralised canteen, toilet location, having colleagues in other offices encouraged movement, facilitating the reduction of sitting time. The reliance on computers to complete work was identified as a barrier to reducing sitting time. Participants believed they would find it difficult to complete computer-based work in a standing position without the use of a SSWD.

\section{Productivity}

Workplace priorities were deemed a barrier to reducing sitting time at work. Participants agreed that a reduction in sitting time was viewed as peripheral to core business. Reducing sitting time whilst maintaining adequate productivity levels was difficult and work demands took priority. The nature of office work was seen to be both a facilitator and a barrier to sitting. Being immersed in work was reported as a barrier to reducing sitting time, where many did not realise how long they were sitting. Some participants found that they needed long periods of time uninterrupted to "get in the zone". Others, however, acknowledged that interrupting sitting throughout the day allowed the opportunity for a "brain break", ultimately leading to better productivity.

\section{Judgemental culture}

The fear of judgement from others acted as a barrier to reducing sitting time. Staff did not want to be seen to be engaging in anything that may be perceived as an unacceptable social or professional norm, for fear of being judged by colleagues. Participants reported the need for a valid reason to be away from their desk. Concerns were raised that reducing sitting time may distract colleagues or draw unwanted attention to themselves. Participants believed that standing may make others feel uncomfortable in certain situations such as team meetings. To increase acceptability, standing at work needed to be "normalised". Participants believed that having approval from employers would have a strong facilitating influence on reducing sitting time and the feeling of being judged. 


\section{Knowledge}

Participants acknowledged a vague awareness of negative health consequences linked to prolonged sitting, but that they lacked good insight. Participants mainly associated prolonged sitting with pain from postural issues. It was this belief that acted as a facilitator to reducing sitting time. Participants were unsure how to reduce occupational sitting time and why this would be beneficial. They believed information on alternatives to sitting would be beneficial. Participants believed that it was sufficient to engage in PA outside the workplace instead of attempting to target a reduction in occupational sitting time. Many participants did not perceive occupational sitting as problematic believing the interruption of sitting for bathroom/tea breaks etc. was sufficient to offset negative health consequences.

Many participants expressed concern that standing may cause discomfort and held doubts regarding the benefits of replacing sitting with standing, as opposed to engaging in light PA. There was a consensus that more understanding by stakeholders such as employers, directors or board members may help to reduce sitting time and an evidence-based business case covering employee health, productivity and absenteeism was believed to be required to obtain their support.

\section{Suggested non technology-supported strategies}

The non technology-supported strategies suggested by participants were categorised into five approaches: 1. Environmental Approach, 2.Workday Approach, 3. Organisational Approach, 4. Educational Approach and 5. Team Approach. These are detailed in Table 2.

Environmental Approach: Participants suggested strategies such as SSWDs, centralised facilities e.g. printers, coffee machines, open plan office, portable IT equipment and active sitting furniture e.g. desk pedal exerciser. These suggestions were met with concern that new equipment may not be used after the initial novelty period. Lack of space, high cost associated, and cabling issues for IT equipment were also noted for consideration. There also were beliefs that working at standing height may be visual invasion/distraction to others. 
Workday Approach: Participants suggested ways to incorporate sitting breaks into normal day to day work routine. Suggestions included scheduling standing/walking meetings, incorporate standing/moving into work tasks e.g. while on phone and using lunch time/coffee breaks as an opportunity to engage in PA. These suggestions allowed movement to be purposive where possible so as not to affect productivity

Organisational Approach: Participants suggested having a managerial "sit-less" role model in the office would be an effective strategy. Flexible working hours to allow for PA during the workday was also suggested. It was noted that strategies would benefit from having support and approval by managers and boards of directors

Educational Approach: Strategies suggested included distribution of information regarding the issues prolonged sitting at work such as educational posters and office health checks. Participants suggested that using "shock tactics" relating to the health risks linked to prolonged sitting as an effective method of disseminating information and capturing attention.

Team Approach: Office competitions were mentioned as an opportunity to increase motivation to participate in an occupational "sit less" initiative. This may increase participation and enhance engagement. Tapping into people's competitive streaks and creating a social element may be a useful method. There were concerns that this competition may draw unwanted attention to those at top and bottom of the scoreboard.

INSERT TABLE 2 HERE

\section{Suggested technology-supported strategies}

The participant suggested technology-supported strategies were categorised into two approaches: 1. Digital reminders and prompts and 2. Activity/sitting time monitoring. These are detailed in Table 3.

Digital reminders and prompts: These were suggested as a mechanism to draw attention to long periods of sitting and encourage breaks, however they may be ignored. Suggested 
approaches were via a mobile app, computer software, wearable device and emails. Some believed that these reminders may become irritating, distracting or cause stress, and therefore the frequency and timing of prompts must be considered. While others agreed that these prompts may be an acceptable way to interrupt a period of sitting with other activities in a structured fashion, others were concerned that they may appear unprofessional in an occupational setting.

Activity/sitting time monitoring: It was suggested that using apps and wearables e.g. activity trackers to self-monitor sitting time/PA may allow users to become more aware of sitting time, set goals, receive tailored feedback, track progress and compete with other users. Some participants were concerned that people may become obsessed with tracking behaviours. Although they were seen to be "trendy", the feasibility of these strategies was met with concern that the devices were costly and may not be used long-term. Participants preferred devices that were non-bulky, portable, easy to use and required minimal interaction.

Overall, technology-supported strategies were highlighted as a valuable approach. Low burden interventions, delivered in a personalised, accurate and non-patronising fashion were preferred. It was suggested that when using technology-supported interventions the type of worker, the tasks involved and their personal preferences should be considered. The potential for these approaches to trigger negative emotional reactions, such as guilt, by potentially highlighting high levels of sitting or low levels of PA or failure to meet set goals was highlighted. Participants were generally not concerned about their employer having access to employee activity data as long as it was used to improve health. It was acknowledged that there was no ideal "one size fits all" approach.

INSERT TABLE 3 HERE

\section{Discussion}

This study revealed barriers, facilitators and potential strategies to reducing occupational sitting. The primary result was that while at work, work tasks were the main priority. Therefore, initiatives to reduce sitting time may come secondary to job tasks and this 
prioritisation of work should be considered in sitting time reduction interventions. Other barriers included "sitting-centred" office design, reliance on IT, fear of being judged as avoiding work when not sitting, and a lack of knowledge on how and why to reduce sitting time. Facilitators to reducing prolonged sitting included open-plan offices with centralised facilities and standing-friendly furniture, "brain breaks", feeling it is acceptable amongst colleagues and managers to be away from your desk, having reason within your work day to be away from your desk e.g. visiting a colleague's office, relief of musculoskeletal pain and evidence-based business cases to reduce sitting time. Potential strategies such as SSWDs, education on the negative health consequence and ways to reduce sitting time, flexible workplace policies and active breaks were suggested. Technology-supported strategies such as apps, computer software, wearable devices and emails were generally seen to be useful tools. Participants preferred low burden interventions, that caused little disturbance, and delivered in a personalised, accurate and non-patronising fashion. In particular, its use was thought to be most valuable in providing prompts and as a platform to allow behavioural selfmonitoring.

The emergence of "primacy of work" as the overarching theme was an important consideration for researchers and intervention developers when designing "sit-less" interventions. Participants believed that the main focus at work should be on completing the tasks they are paid to do. Concerns have been noted elsewhere that workplace health interventions may distract workers from their duties, negatively impacting productivity. ${ }^{24} 27$ 18 In the current study, there were varying views on how interrupting sitting with other activities may affect productivity, with some believing breaks were detrimental and others noting a positive impact. There is a lack of evidence available on the direct influence of workplace sitting on productivity 242829 and this may be a reason for the conflicting views. This highlights the need for future work to explore links between sitting time breaks and productivity and to use productivity as an outcome measure when assessing intervention efficacy.

Participants in the current study identified aspects of the workplace environment as being both barriers and facilitators to sitting. Healy et al, ${ }^{30}$ suggest that the built environment plays an important part in reducing occupational sitting time and is a key consideration in 
developing occupational "sit-less" programmes. Participants in the current study also felt that standing-friendly furniture was a particularly promising strategy, as they allowed a reduction in sitting time without impacting productivity. Being able to reduce sitting time while getting your job done e.g. active meetings was considered a positive option. This finding is in line with those of Hadgraft et al, ${ }^{24}$ where strategies that have dual benefits may encourage greater uptake and engagement. It is also important to note that a recent systematic review and meta-analysis suggest that SSWDs may be effective in reducing workplace sitting time at short-term and medium-term follow-up. ${ }^{29}$

Another barrier to reducing sitting time was the poor understanding of the negative health consequences of prolonged sitting. This reflects findings from Duncan et $\mathrm{al}^{31}{ }^{31}$ who suggest that two thirds of the adult population are unaware of the associated risks. Participants noted that if they were better informed about the negative health concerns they may face in the future due to prolonged sitting, they may be more motivated to change. Education may work as a strategy by highlighting the potential future negative health consequence associated with a sedentary desk job, thereby motivating participants to change their behaviour. ${ }^{32}$

According to participants in this study, there is potential value in using technology-supported strategies as a platform to self-monitor sitting time to reduce occupational sitting. This is an encouraging finding as self-regulatory techniques, such as self-monitoring, have been shown to be common features in promising sitting time reduction interventions. ${ }^{33}$ Another potentially useful strategy, to reduce sitting time using technology-supported strategies, was prompting users to interrupt sitting with other activities. Technology-supported strategies involving cues to interrupt sitting at work may be particularly useful for office workers as the non-conscious nature of sitting likely limits awareness of true sitting time. ${ }^{33}$

Within the identified strategies, a desire for personal choice was evident. This view has also been noted in other workplace health studies ${ }^{34} 35{ }^{36}$ due to different office-based roles and individual preferences. This suggests that a "one size fits all" approach may not be effective. This has been highlighted elsewhere, highlighting the need for tailored and pragmatic approaches to encouraging a change in sitting and activity in the workplace. ${ }^{34}$ Technologysupported strategies offer a platform to overcome this hurdle as it provides an opportunity 
to personalise intervention content. ${ }^{13}$ Although, having personal choice over intervention strategies was preferred, results from the current study suggest that it may not be enough for an individual to change their sitting behaviour alone. This is highlighted by their suggestions of strategies such as 'team approach' and 'role models'. Due to these conflicting suggestions, it may be that both personal choice and external support structures could be incorporated in the same intervention.

Participants were concerned that prompts may become annoying and disturbing which has also been recognised by Dennison et al. ${ }^{37}$ Participants in the current study also highlighted the potential for technology-supported strategies to trigger negative emotional reactions, such as guilt, by potentially highlighting high levels of sitting or failure to meet set goals. Selfmonitoring technology-supported strategies have previously been linked to evoking feelings of guilt in users ${ }^{38}$ however, this feeling of guilt may be an enabler of motivation and willpower. ${ }^{39}$ These considerations should be considered by future intervention developers in the area. Participants also mentioned distrust of digitally gathered information if it is not accurate. Sanders et al, ${ }^{40}$ suggest that accurate and trustworthy self-monitoring data are required for more potent behaviour change. Results from the current study identified that a successful technology-supported strategies must have low user burden, should not negatively impact upon their work and should provide accurate data. Concerns were raised that if the intervention did not minimise these issues, it could ultimately lead to disengagement. These challenges are widely noted throughout the digital behaviour change literature. ${ }^{37414243}$. At this time, participants in the current study generally did not express concern regarding their employer having access to their activity data. This is a promising finding as it has been noted elsewhere that technology users may be concerned about the privacy and ownership of their health data. ${ }^{40}$

A strength of this study is that it provides insights into the perspectives of target users and stakeholders, whose voices are critical in developing worksite health interventions. ${ }^{19}$ The study also recruited a heterogeneous sample of office workers deemed to be representative of a typical office-based workforce. Using the TDF to structure the topic guide ensured a robust theoretical basis to the questioning that covered a wide range of behavioural influences. ${ }^{26}$ To ensure reliability of coding, analyses were carried out independently by two 
researchers. A limitation of the study is that although sufficient data were collected, recruitment of participants in the employer and board level categories was particularly difficult. Establishing initial contact within the organisation was the largest barrier to recruitment. A potential solution to this issue may be via the wider education of all employers to the benefits of a reduction in employee occupational sitting time.

\section{Conclusions}

To our knowledge, this study was the first to investigate perceptions of employees, employers and board members, regarding the use of technology-supported strategies as a potential tool to reduce workplace sitting time. The findings provide insights into the barriers, facilitators, strategies and key considerations that should be given attention when designing interventions to tackle occupational sitting. It was identified that above all, interventions should not impact upon the primacy of work. Intervention designers need to consider individual preferences, as well as the environmental factors, the judgemental culture, productivity concerns and the knowledge levels of employees and employers. Technologysupported strategies such as apps, computer software, wearable devices and emails were generally seen to be useful tools. They were thought to be particularly valuable in providing prompts and as a platform to allow behavioural self-monitoring. The lessons gained from this qualitative work may play an important part in future intervention design. 
References

1. Parry S, Straker L. The contribution of office work to sedentary behaviour associated risk. BMC Public Health 2013;13. doi:10.1186/1471-2458-13-296

2. Clemes S, Patel R, Mahon C et al. Sitting time and step counts in office workers. Occup Med 2014;64:188-192. doi:10.1093/occmed/kqt164

3. Kazi A, Duncan $\mathrm{M}$, Clemes $\mathrm{S}$ et al. A survey of sitting time among UK employees. Occup Med 2014;64:497-502. doi:10.1093/occmed/kqu099

4. Waters $\mathrm{C}$, Ling $\mathrm{E}, \mathrm{Chu} \mathrm{A}$ et al. Assessing and understanding sedentary behaviour in office-based working adults: a mixed-method approach. BMC Public Health 2016;16. doi:10.1186/s12889-016-3023-z

5. Hadgraft N, Healy G, Owen N et al. Office workers' objectively assessed total and prolonged sitting time: Individual-level correlates and worksite variations. Prev Med Rep 2016;4:184-191. doi:10.1016/j.pmedr.2016.06.011

6. Rezende L, Rodrigues Lopes M, Rey-López J et al. Sedentary Behavior and Health Outcomes: An Overview of Systematic Reviews. PLoS ONE 2014;9:e105620. doi:10.1371/journal.pone.0105620 
7. Biswas A, Oh P, Faulkner G et al. Sedentary Time and Its Association With Risk for Disease Incidence, Mortality, and Hospitalization in Adults. Ann Intern Med 2015;162:123. doi:10.7326/m14-1651

8. Ekelund U, Steene-Johannessen J, Brown W et al. Does physical activity attenuate, or even eliminate, the detrimental association of sitting time with mortality? A harmonised meta-analysis of data from more than 1 million men and women. Lancet 2016;388(10051):1302-1310.

9. Pandey A, Salahuddin U, Garg S et al. Continuous Dose-Response Association Between Sedentary Time and Risk for Cardiovascular Disease. JAMA Cardiol 2016;1(5):575.

10. van Uffelen J, Wong J, Chau J et al. Occupational Sitting and Health Risks. Am J Prev Med 2010;39:379-388. doi:10.1016/j.amepre.2010.05.024

11. Schmid D, Leitzmann M. Television Viewing and Time Spent Sedentary in Relation to Cancer Risk: A Meta-Analysis. JNCI 2014;106. doi:10.1093/jnci/dju098

12. Straker L, Coenen P, Dunstan D et al. Sedentary Work - Evidence on an Emergent Work Health and Safety Issue. Canberra. Safe Work Australia. 2016. https://www.safeworkaustralia.gov.au/doc/sedentary-work-evidence-emergentwork-health-and-safety-issue (accessed 21 Jan 2018). 
13. West R, Michie S. A guide to development and evaluation of digital behaviour interventions in healthcare. Silverback Publishing 2016

14. Brakenridge C, Fjeldsoe B, Young D et al. Evaluating the effectiveness of organisational-level strategies with or without an activity tracker to reduce office workers' sitting time: a cluster-randomised trial. IJBNPA 2016;13. doi:10.1186/s12966-016-0441-3

15. Evans R, Fawole H, Sheriff S et al. Point-of-Choice Prompts to Reduce Sitting Time at Work. Am J Prev Med 2012; 43:293-297. doi:10.1016/j.amepre.2012.05.010

16. Arrogi A, Bogaerts A, Seghers J et al. Evaluation of stAPP: a smartphone-based intervention to reduce prolonged sitting among Belgian adults. Health Promot Int 2017;34:16-27. doi:10.1093/heapro/dax046

17. Stephenson A, McDonough S, Murphy M et al. Using computer, mobile and wearable technology enhanced interventions to reduce sedentary behaviour: a systematic review and meta-analysis. IJBNPA 2017;14. doi:10.1186/s12966-0170561-4

18. Rennekamp R, Nall N. Using Focus Groups in Program Development and Evaluation. University of Kentucky 2006. https://psd.ca.uky.edu/files/focus.pdf (Accessed 1 Feb. 2017) 
19. van Berkel J, Meershoek A, Janssens R et al. Ethical considerations of worksite health promotion: an exploration of stakeholders' views. BMC Public Health 2014;14. doi:10.1186/1471-2458-14-458

20. Wierenga $D$, Engbers $L$, Van Empelen $P$ et al. What is actually measured in process evaluations for worksite health promotion programs: a systematic review. BMC Public Health 2013;13. doi:10.1186/1471-2458-13-1190

21. Deliens T, Deforche B, De Bourdeaudhuij I et al. Determinants of physical activity and sedentary behaviour in university students: a qualitative study using focus group discussions. BMC Public Health 2015;15. doi:10.1186/s12889-015-1553-4

22. De Cocker K, Veldeman C, De Bacquer D et al. Acceptability and feasibility of potential intervention strategies for influencing sedentary time at work: focus group interviews in executives and employees. IJBNPA 2015;12. doi:10.1186/s12966-015-0177-5

23. Cole J, Tully M, Cupples M. “They should stay at their desk until the work's done": a qualitative study examining perceptions of sedentary behaviour in a desk-based occupational setting. Implement Sci 2015;8. doi:10.1186/s13104$015-1670-2$ 
24. Hadgraft N, Brakenridge C, LaMontagne A et al. Feasibility and acceptability of reducing workplace sitting time: a qualitative study with Australian office workers. BMC Public Health 2016;16. doi:10.1186/s12889-016-3611-y

25. Cane J, O'Connor D, Michie S. Validation of the theoretical domains framework for use in behaviour change and implementation research. Implement Sci 2012;7. doi:10.1186/1748-5908-7-37

26. Atkins L, Francis J, Islam R et al. A guide to using the Theoretical Domains Framework of behaviour change to investigate implementation problems. Implement Sci 2017;12. doi:10.1186/s13012-017-0605-9

27. Braun V, Clarke, V. Thematic analysis. In H. Cooper, P. M. Camic, D. L. Long, A. T. Panter, D. Rindskopf, \& K. J. Sher (Eds.), APA handbook of research methods in psychology, Vol. 2. Research designs: Quantitative, qualitative, neuropsychological, and biological (pp. 57-71). Washington, DC, US: American Psychological Association.

28. Gilson N, Straker L, Parry S. Occupational sitting: practitioner perceptions of health risks, intervention strategies and influences. Health Promot J Aust 2012;23:208-212. doi:10.1071/he12208 
29. Shrestha N, Kukkonen-Harjula K, Verbeek J, ljaz S, Hermans V, Pedisic Z. Workplace interventions for reducing sitting at work. Cochrane Database of Systematic Reviews. 2018;20(6).

30. Healy G, Lawler S, Thorp A et al. Reducing prolonged sitting in the workplace (An evidence review: full report). 2012. https://www.sacoss.org.au/sites/default/files/public/documents/Reducing_Sittin g_Workplace_Summary.pdf (accessed 14 Aug 2017).

31. Duncan M, Gilson N, Vandelanotte $\mathrm{C}$. Which population groups are most unaware of CVD risks associated with sitting time?. Prev Med 2014;65:103-108. doi:10.1016/j.ypmed.2014.05.009

32. Tasdemir-Ozdes A, Strickland-Hughes $C$, Bluck S et al. Future perspective and healthy lifestyle choices in adulthood. Psychol Aging 2016;31:618-630. doi:10.1037/pag0000089

33. Gardner B, Smith L, Lorencatto $\mathrm{F}$ et al. How to reduce sitting time? A review of behaviour change strategies used in sedentary behaviour reduction interventions among adults. Health Psychol Rev 2015;10:89-112.

doi:10.1080/17437199.2015.1082146 
34. Gilson N, Burton N, van Uffelen J et al. Occupational sitting time: employees' perceptions of health risks and intervention strategies. Health Promot J Aust 2011;22:38-43. doi:10.1071/he11038

35. Tsiga E, Panagopoulou E, Niakas D. Health promotion across occupational groups: one size does not fit all. Occup Med 2015;65:552-557. doi:10.1093/occmed/kqv097

36. Stephens S, Eakin E, Clark B, Winkler E, Owen N, LaMontagne A et al. What strategies do desk-based workers choose to reduce sitting time and how well do they work? Findings from a cluster randomised controlled trial. IJBNPA 2018;15(1).

37. Dennison L, Morrison L, Conway G et al. Opportunities and Challenges for Smartphone Applications in Supporting Health Behavior change: Qualitative Study. JMIR 2013;15:e86. doi:10.2196/jmir.2583

38. Sjjklint M, Constantiou I, Trier M. The Complexities of Self-Tracking - An Inquiry into User Reactions and Goal Attainment. SSRN E Journal 2015. doi:10.2139/ssrn.2611193

39. Hoch S, Loewenstein G. Time-Inconsistent Preferences and Consumer SelfControl. J Consum Res 1991;17:492. doi:10.1086/208573 
40. Sanders J, Loveday A, Pearson N et al. Devices for Self-Monitoring Sedentary Time or Physical Activity: A Scoping Review. JMIR 2016;18:e90. doi:10.2196/jmir.5373

41. Eysenbach G. The Law of Attrition. JMIR 2005;7:e11. doi:10.2196/jmir.7.1.e11

42. Vandelanotte C, Kolt G, Caperchione C et al. Effectiveness of a Web 2.0 Intervention to Increase Physical Activity in Real-World Settings: Randomized Ecological Trial. JMIR 2017;19(11):e390.

43. Carolan S, de Visser R. Employees' Perspectives on the Facilitators and Barriers to Engaging With Digital Mental Health Interventions in the Workplace: Qualitative Study. JMIR Ment Health 2018;19(5):1. 
